\title{
LETTERS
}

\section{Alarming Ontario drinking statistics obligate reappraisal of the impact of alcohol on newborns}

The recent study by Myran and colleagues on rates of emergency department visits attributable to alcohol use $\mathrm{e}^{1}$ is among the most comprehensive accounts of alcohol abuse in Ontario. The results show that from 2003 to 2016, emergency department visits for alcohol consumption increased 4.4 times more than overall visits.

The study also identifies a number of relevant demographic features. First, the increase in visits to the emergency department is greater for women (86.5\%) than for men (53.2\%), with 25- to 29-year-olds showing the largest (175\%) increase. Second, the highest rate of emergency department visits attributed to alcohol abuse were for 15- to 24-year-old women and 45- to 54-year-old men. Third, among the medical harms identified was suspected fetal damage, including fetal alcohol syndrome, which increased from 6 cases in 2003 to 134 in 2016. This represents an increase of $2133.3 \%$, the largest percentage increase in any of the health outcomes assessed (range 3.7\% increase for alcohol dependence to $245.5 \%$ increase for alcohol-related mental and behavioural disorders).

Given the nature of the data used in this report, these numbers are likely an underestimate and include only the extreme cases. This trend documented for 2003 and 2016 for Ontario must be viewed as multifactorial. However, the conclusion that young women of reproductive age represent a segment of society that is increasingly attracted to alcohol use is unavoidable. In doing so, this group exposes themselves - and the fetus in cases of recognized or unrecognized pregnancies - to the negative health effect of alcohol. Often, alcohol-exposed pregnancies can lead to fetal alcohol spectrum disorder (FASD). ${ }^{2}$

Fetal alcohol spectrum disorder is a serious neurodevelopmental disorder. It is caused exclusively by prenatal alcohol exposure from maternal drinking. There is no known safe dose of alcohol or safe timing of alcohol exposure during pregnancy. ${ }^{3}$ Most of the defects we see in FASD represent the tip of the iceberg. Diagnosis of FASD is subjective. There is no biological diagnostic test, and there is no cure.

Given the alarming increase in alcohol consumption ( $175 \%$ ) by young women of reproductive age seen in this study ${ }^{1}$ and the apparently high incidence of FASD in Ontario (about 3\%), ${ }^{4}$ alcohol consumption demands concerted action. In the long term, this action must focus on research to understand the mechanism of action of alcohol on fetal development and how to reduce its impact. ${ }^{5}$ Immediately and at a minimum, there is an urgent need and obligation to take measures to reduce alcohol abuse, particularly in high-risk groups.

Ours is an alcohol-friendly society. Alcohol is easily accessible, commonly used and advertised. Although highly addictive, alcohol is part of our social fabric. As identified in the report by Myran and colleagues, alcohol use represents a major impediment to societal health and well-being. ${ }^{1}$ These results suggest that current regulations of the use and abuse of alcohol are not effective, and measures by government, industry and society that will offer checks and balances to its harms are needed.

On the surface, the issue of FASD appears straightforward. No maternal drinking during pregnancy means no FASD and related disorders. However, in practice it has been hard to prevent alcohol use during pregnancy. Educational programs, including youth education at home and in schools, are needed to reinforce the message of "no alcohol during pregnancy." Doing nothing is not an option.

\section{Bonnie Alberry BSc}

PhD candidate, Western University, London, Ont.

\section{Shiva M. Singh PhD}

Distinguished university professor emeritus, Western University, London, Ont.

- Cite as: CMAJ 2019 November 18;191: E1283. doi: 10.1503/cmaj.73305

\section{References}

1. Myran DT, Hsu AT, Smith G, et al. Rates of emergency department visits attributable to alcohol use in Ontario from 2003 to 2016: a retrospective population-level study. CMAJ 2019;191:E804-10.

2. Sokol RJ, Delaney-Black V, Nordstrom B. Fetal alcohol spectrum disorder. JAMA 2003;290:2996-9.

3. Singh SM, Laufer BI, Kapalanga J. Fetal alcohol and the right to be born healthy .... Front Genet 2014;5:356.

4. Popova S, Lange S, Chudley AE, et al. World Health Organization international study on the prevalence of fetal alcohol spectrum disorder (FASD): Canadian component. Toronto: Centre for Addiction and Mental Health; 2018

5. Chokroborty-Hoque A, Alberry B, Singh SM. Exploring the complexity of intellectual disability in fetal alcohol spectrum disorders. Front Pediatr 2014;2:90.

Competing interests: None declared. 